\title{
LIQUID CHROMATOGRAPHIC ANALYSIS OF CAROTENOIDS IN FOODS
}

\author{
DEMET DINCEL ${ }^{1^{*}}$, SERIFE EVRIM KEPEKCI TEKKELI ${ }^{2}$, CEM ÖNAL ${ }^{3}, A R M A \breve{G A N} O ̈ N A L^{3}$, OLCAY SAGIRLI ${ }^{3}$ \\ 1 Bezmialem Vakif University Faculty of Pharmacy Department of Pharmacognosy 34093, Fatih, Istanbul, Turkey \\ 2 Bezmialem Vakif University University Faculty of Pharmacy Department of Analytical Chemistry 34116, Fatih, Istanbul, Turkey \\ 3 Istanbul University Faculty of Pharmacy Department of Analytical Chemistry 34116, Beyazit, Istanbul, Turkey
}

\section{ABSTRACT}

Current scientific studies indicate that carotenoids (CRTDs) are beneficial for maintaining health and lowering the risk of different pathologies. Considering the increasing role of carotenoids in healthcare, determination of carotenoid content of foods become important. Mostly, the analytical procedures that are used for this aim, are based on extraction techniques followed by different analytical procedures, especially high performance liquid chromatography. In this publication, a review of various analytical techniques developed for the determination of carotenoids in different food commodities starting from 2006 up to now, has been presented.

Abbrevations: CRT,Carotene; CRTD, Carotenoid; AA, Ascorbic acid; LT, Lutein; CRX, Cryptoxanthin; ZX, Zeaxanthin; HPLC; High Performance Liquid Chromatography; DAD, Diode Array Detector; PDA, Photodiode Array; UV, Ultraviyolet spectroscopy; LC-MS, Liquid Chromatograhy-Mass Spectroscopy; APCIMS, Atmospheric Pressure Chemical Ionization Mass Spectrometry; DIDA, Diastereomeric Dilution Assay; HF-LPME, Hollow Fiber Liquid-phase Microextraction; UPLC, Ultra Performance Liquid Chromatography; GC-MS, Gas Chomatography-Mass Spectroscopy; ELSD, Evaporative Light Scattering Detection; TQMS, TripleQuadrupole Mass Spectroscopy;

Keywords: CRTs, High-performance Liquid Chromatography, Food analyses, Extraction.

\section{INTRODUCTION}

According to epidemiological studies, carotenoids (CRTDs) are the pigments found in higher plants, vegetables and fruits [1]. Traditionally, CRTDs have been commonly used in foodstuffs as colorants. Latest researchs revealed the several important roles of CRTDs, specifically in its antioxidant activity [2], its preventive and curing effects on many disease like cardiovascular diseases [3], cancer [4] and macular degeneration [5]. Different plants and microorganisms are responsible for the biological synthesis of CRTDs as natural pigments. Animals are not able to synthesize CRTDs and they are described as hydrocarbons [carotenes (CRT)] and have hydrophobic properties. Oxygenated derivatives of CRTDs are named as xanthophylls, which have 8 isoprenoid units combined and the configuration of isoprene units is inverted at the origin of the molecule thereby the two centric methyl groups are in 1,6-configurational relationship and the rest of nonterminal methyl groups are related to 1,5 configuration. The whole of CRTD compounds are able to synthesized starting from the acyclic pioneer, having a length centric chain of conjugated double linkages [enzymatically synthetised by involvement of one of the following enzymatic procedurs or a combination of them] a) hydrogenated, b) dehydrogenated, c) cyclization, d) oxidation [6].

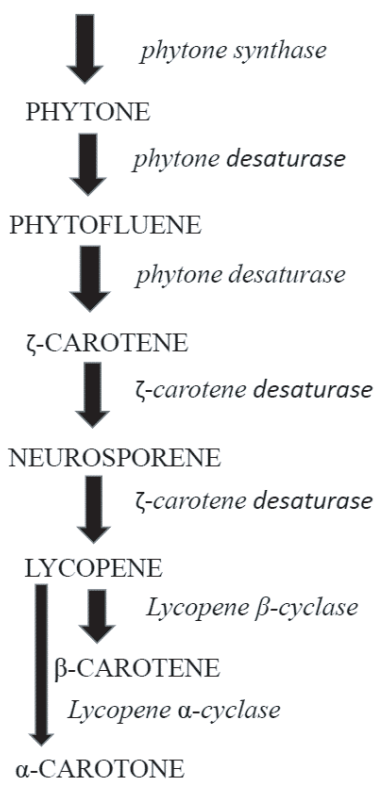

Figure 1: Schematic diagram of the biosynthetic pathway of carotenoids in plants.
Following the first structural identification of $\beta$-CRT, a number of CRTD compounds were reported in natural resources and are discovered every year. Various plants, algae and photosynthetic bacteria synthesize CRTD through biological/enzymatic pathways [7]. The sequences of biosynthesis of CRTDs in plants is described in Fig.1. Enzymatic process starting from phytoene leading to lycopene (LYC), which is a spherical molecule having 13 double bonds. The enzymatic process following LYC involves cyclization of the end groups, leading to the formation of $\alpha$-CRT (one beta ring) and $\beta$-CRT (two beta rings). Xanthophylls are biosynthesized with the addition of oxygen to the molecule [8]. The essential sources of major CRTD compounds are listed in Table 1.

Table 1: The main sources of some carotenoids

\begin{tabular}{|l|l|}
\hline Carotenoid & Source \\
\hline Lycopene & $\begin{array}{l}\text { Tomato and tomato products, dried apricots, } \\
\text { guava, watermelon, papaya and pink grapefruit, }\end{array}$ \\
\hline $\boldsymbol{\beta}$-carotene & $\begin{array}{l}\text { Carrots, Broccoli, Red pepper, Spinach } \\
\text { Apricots, sweet potatoes, green leafy vegetables, } \\
\text { orange and yellow fruits and vegetables, pumpkin, } \\
\text { peach fruits, juice, jams, grapefruit, tomato fruit, } \\
\text { oil seed crops of peanut, soybean, sunflower and } \\
\text { mustard, pepino fruit, egg yolk, tomato leaves and } \\
\text { fruits, freshwater green algae, leafy vegetables, } \\
\text { poultry feed }\end{array}$ \\
\hline $\boldsymbol{\alpha}$-carotene & Carrots, Corn, Cloudberry, pepino fruit \\
\hline Lutein and zeaxanthin & $\begin{array}{l}\text { spinach and kale, pumpkin, peach fruits, juice, } \\
\text { jams, tomato fruit, egg yolk, poultry feed }\end{array}$ \\
\hline
\end{tabular}

Epidemiological human studies revealed that diet with low CRTD content may be correlated with remained hazard for cancer, age-related macular degeneration, cataract, skin damage from sun-burn or cardiovascular disorders [9]. Because of these data, the CRTD content of food products gained high importance. Numerous analytical methods have been improved for identification of CRTD in different kinds of foods. Since 2005, liquid chromatographic methods have been reviewed for the detection of CRTDs in different food products

\section{ANALYTICAL METHODS}

HPLC has been applied as an effective analytical method for quantitative analysis of low amounts of CRTDs in foods.

The most preferred methods for CRTDs analysis are based upon ultraviyolet (UV) -visible and diode array (DAD) detections. LYC and $\beta$-CRT constituents of vegetables including tomato, medlar, carrot, pepper, watermelon, persimmon were determined by a HPLC method [10]. Direct extraction was performed in one step using hexane/acetone/ethanol mixture. 
Provitamin A and retinol in milk and dairy products were determined simultaneously by a validated HPLC method after all the extraction of fat, pursued by slight saponification. Retinoid and CRTD content per 100 gram of raw milk (4.4\% fat), overall fat milk (standardized to $3.5 \%$ fat), low-fat milk (standardized to $1.5 \%$ fat) are as follows respectively; $20 \mu \mathrm{g}$ CRTDs and $40 \mu \mathrm{g}$ retinol for raw milk, $34 \mu \mathrm{g}$ retinol and $18 \mu \mathrm{g}$ CRTD for full fat milk; $14 \mu \mathrm{g}$ retinol and $9 \mu \mathrm{g}$ CRT for low-fat milk [11]. For determination of 3R,3'-Rzeaxanthin $(\mathrm{ZX})$ in plant derived food such as corn, deep-frozen spinach, orange pepper, wheat flour, a DIDA using chiral HPLC-DAD assay was improved [12], internal standard was diastereomeric, 3R,3'-S-(meso)- ZX. Based on the recovery rate of $94.7 \%$ and reliable results; DIDA was found to be a valuable analytical tool especially when coupled with saponification.

Quantification methods for CRTDs using HPLC and screening of CRTD with spectroscopy were developed by Kimura et.al. [13]. Concentrations of $\alpha-$ and $\beta$ - CRT, cryptoxanthin (CRX), LYC, lutein (LT) and ZX were quantified in ten varieties of 5 fruit species; orange, cherry peach, apple, pear and 5 varieties of 4 species of vegetables; purslane, Portuguese coles, turnip greens, beetroot leaves and leaf beet cultivated in Portugal and country conventional. The leafy vegetables were identified as a rich content of $\beta$-CRT $(0.46-6.4 \mathrm{mg} / 100 \mathrm{~g})$; LT $(0.52-7.2 \mathrm{mg} / 100 \mathrm{~g})$ despite the fruits are found to have a relatively small amount of CRTDs (LT, $0.0032-0.16 \mathrm{mg} / 100 \mathrm{~g}$ and $\beta$-CRT, $0.010-0.17 \mathrm{mg} / 100 \mathrm{~g}$ ). 8 varieties of citrus fruits have been analysed for the content of main CRTDs for examples LT, ZX, $\beta$-CRX, and $\beta$-CRT [14]. After extraction with hydroxytoluene, a HPLC method was used. $\beta$-CRX was identified as the major CRT $(0.764 \pm 0.031-6.67 \pm 0.329 \mu \mathrm{g} / \mathrm{g} \mathrm{db})$, pursued by $\beta$-CRT $(0.435 \pm 0.016-$ $3.77 \pm 0.154 \mu \mathrm{g} / \mathrm{g} \mathrm{db}$ ). CRTD content covering LT, ZX, LYC, $\beta$-CRX, $\alpha$ - and $\beta$-CRT was quantified in six different species of berry blackberry, blueberry, raspberry, strawberry, red and black currant [15].

All CRTD context was to be discovered the highest in blackberry (440 $\mu \mathrm{g}$ / $100 \mathrm{~g})$, pursued by raspberry $(370 \mu \mathrm{g} / 100 \mathrm{~g})$. The lowest content of CRTD was detected with red currant $(45 \mu \mathrm{g} / 100 \mathrm{~g})$ and strawberry $(26 \mu \mathrm{g} / 100 \mathrm{~g})$. LT was determined in milk powders, human milk, bovine, baby formulas by HPLC method after alkaline hydrolysis in a single-tube and one step extraction with hexane:di isopropyl ether $(75: 25 ; \mathrm{v} / \mathrm{v})$ [16]. After reconstitution of infant formulas and bovine milk powder, endogenous LT levels were found to be in the range of 1.0 to $5.8 \mu \mathrm{g} / 100 \mathrm{~mL}$. LT levels in bovine and human milk were found as 6.0 and $4.3 \mu \mathrm{g} / 100 \mathrm{~mL}$, alternately. CRTD ingredient of fruits and vegetables cultivated in Portugal have been qualitatively and quantitavely determined with a HPLC method using a tomato food matrix by in-house validation, testing sample and standards stability, appreciating measurement uncertainity and contributing to sampling planning [17]. CRTD concentration and measurement were, alternately, $1.0 \pm 0.14 \mathrm{mg} / 100 \mathrm{~g}$ and $0.39 \pm 0.056 \mathrm{mg} / 100 \mathrm{~g}$ for trans-bCRT, $8 \pm 2.0$ and $2.3 \pm 0.57$ for all-LYC, $0.10 \pm 0.017$ and $0.08 \pm 0.015$ for LT; trans-a-CRT, b-CRX and ZX were not determined in both species.

Serino et.al. [18] determined simultaneously four major CRTD using microextraction technique and HPLC. N-hexane, sodium chloride dichloromethane and ethyl acetate were used for microextraction of CRTD from fresh tomato powder. Microextraction method avoids evaporation step and it is applicable to small size samples which provide greater advantages compared to other extraction methods. LT and ZX context of some vegetable oils and vegetables extensively used in the Indian diet were identified by a HPLC method [19]. LT levels were found to be the highest in pumpkin $(10,620 \mu \mathrm{g} / 100 \mathrm{~g})$ pursued by chilli $(1900 \mu \mathrm{g} / 100 \mathrm{~g})$ and brinjal $(1800 \mu \mathrm{g} / 100 \mathrm{~g})$. LT level was the highest in mustard $(772 \mu \mathrm{g} / 100 \mathrm{~g})$ and palm $(11.55 \mu \mathrm{g} / 100 \mathrm{~g})$ oils, LT was not assigned in sunflower, almond, olive, rice bran and corn oils. Taiwanese sweet potatoes harvested at different times were analyzed for cis- and trans alpha and $\beta$-CRTD content by an optimized HPLC method [20]. According to results, the orange-pulp sweet potato (Tainung 66) had high amounts of CRTD context than the yellow-pulp one (Tainung 57) at the same crop time. The whole constituent of CRTD in both yield harvested at different times were higher in the following order of harvest time; 1.) October, 2.) July, 3.) April, 4.) January, respectively. Three HPLC assays for determination of following CRTD in nourishment items were evaluated; $\alpha$ - and $\beta$-CRT, $\beta$-CRX, LT, LYC, and ZX [21]. The most technically suitable and cost effective method is described as follows; extraction using methanol and tetrahydrofuran from food item pursued by evaporating and saponification (if needed) using pyrogallol, implementing a HPLC technique using a dilute gradient mobile phase based upon acetonitrile and a stationary phase consisting of two serial attached C-18 columns. LT, LYC and $\beta$-CRT in tomato and its products were determined by a HPLC method developed by
Huang et.al. [22]. Tomato epicarp was extracted with ethyl acetate by shaking in a water bath with thermostatic shaker for $60 \mathrm{~min}$. Severance of the all-trans and cis isomers of LYC was performed. CRTD and vitamin E content of different oils in the market six rapeseed oils, six sunflower oils (half of each cold pressed), rape oil, sunflower oil, flax oil and safflower oil and also press cakes and the respective seeds from a local oil mill were determined by HPLC. In two cold pressed rapeseed oils, the whole CRTD context varied between $0.05-1.52 \mathrm{mg} /$ $100 \mathrm{~g}$ oil (traces of (all-E)- $\beta$-CRT determined. The safflower oil contained only traces of CRTDs; in the flax oil the content of (all-E)-LT and CRTDs was around $0.4 \mathrm{mg} / 100 \mathrm{~g}$ [23].

A rapid and convenient HPLC assay for the routine qualitative and quantitative detection of CRTDs and fat-soluble compounds from complex matrices was developed by Gleize et.al. [24]. Proposed method is applicable to complex matrices such as fruit, kiwi, spinach, human plasma and human adipose tissue and provide the assay of 11 CRTD, retinol, $\alpha$ - and $\gamma$-tocopherol (TOC) within $35 \mathrm{~min}$. The same method is also applicable for separation of coenzyme Q 10. LYC content of a large scale of food commodities for instance fresh vegetables, fruits and also treated foods were detected with HPLC/UV method [25]. The largest quantity of LYC was determined in tomato and tomatocontaining products. According to study results, level of all-E-LYC was $94 \%$ in raw foods while all-E-LYC content of treated foods (for instance pizza, soups and pasta sauces) was found to be in the range of $76 \%$ to $87 \%$. LT analysis was performed by HPLC using DAD. According to study results, content of LT extracted from various tinning industries tomato seed and peel examples varied between 9.9-10.5 $\mu \mathrm{g} / \mathrm{g}$ dry weight [26].

Content of phylloquinone (PHQ), ascorbic acid (AA), CRTDs and TOCs in 25 trademarks available microgreens were determined by Xiao et.al. [27]. $\alpha$-TOC and $\gamma$-TOC concentration were found to be within the range of 4.9 to 87.4 and 3.0 to $39.4 \mathrm{mg} 100 \mathrm{~g}-1$, individually, meanwhile $\beta$-CRT, LT/ZX and violaxanthin concentrations varied between $0.6-12.1,1.3-10.1$ and $0.9-7.7 \mathrm{mg} / 100 \mathrm{~g}$, successively. Amongst the 25 microgreens, green daikon radish, amaranth, red cabbage, garnet, cilantro had the greatest amounts of AAs, CRTDs, PHQ and TOCs, individually.

The direct analysis of LT in spinach was achieved with different extraction solvents with HPLC method by Simonovska et. al. [28]. The quantity of LT changes between $5-15 \mathrm{mg} / 100 \mathrm{~g}$ of fresh weight of different spinach samples. Various extraction solvents such as acetone, ethanol, methanol-THF 1:1 (v/v), ethanol-ethyl acetate 1:1 (v/v) have been used. Recovery of LT has been increased with an addition of TEAA buffer. The determination of LT, LYC and $\beta$-CRT was performed with using a RP-HPLC assay by Ligor et.al. [29]. In this technique, different types of stationary phases $(\mathrm{C} 18, \mathrm{C} 30)$ were compared to each other and also the addition of trimethylamine in mobile phase showed that impressing the CRTD retention behaviours.

A quick HPLC method was developed by Gupta et.al. [30] for CRT from different part of plant tissues. It is based on using various extraction solvents (diethyl ether, chloroform, dichloromethane, acetone, methanol) with different ratio for analysis of CRTD. Because of the fact that it does not include methanol, the dichloromethane: chloroform $(1: 2)$ mixture gives the best result, by eliminating the interfering metabolites in detection of CRTDs. And also methanol and methyl-tert-butyl ether were used for mobil phase solvents with different ratio. The reason of usage of two varied ratio was related to diversity in constitution of CRTDs in red or green tissues.

The TOC constitution was investigated in different apple seed oils with RPHPLC/FID by Gornas [31]. The method was focused on apples which are grown in different region because of their place of cultivation is important for tocopherol detection. Four $(\alpha, \beta, \gamma$ and $\sigma)$ TOC homologues were analysed and according to the results, seed oils obtained from apples farmed in eastern europe are very substantial resources of TOCs, primarily $\alpha$ - and $\beta$-TOC.

Pro-vitamin A CRTs were determined with a quick HPLC method from four oilseed crops (soybean, sunflower, peanut and mustard) by Bhatnagar-Pabwar et.al. [32]. Analysis of A-CRTD has been difficult because of strong affiliation to oil and their low recovery. In this technique, absolute ethanol was chosen for extraction solvent containing of very polar: non polar ratios. Compared to previous literature, CRTD were detected different protocols for extraction. And the results show that, the detection susceptibility was dramatically higher where the $\beta$-CRT levels as low as $60 \mathrm{ng} / 100 \mathrm{mg}$ could be determined using this method. 
CRTDs, three organic acids (citric, ascorbic, and malic acids) and sugars (Sucrose, glucose, and fructose) were determined using HPLC method from pepino fruits and fruit juices by Kola et.al. [33]. The purpose of this experiment was to analyze the nutrient quality of pepino fruits through its maturation stages. The CRTD composition (xantophyll, ZX, $\beta$-apo- 8 -carotenal, $\alpha$-CRX, $\beta$-CRX, $\alpha$ CRT and $\beta$-CRT) was also investigated from different colors of pepino fruits in maturation period. LT and $\beta$-CRT in egg yolk were analyzed by HPLC-DAD with vortex-mixing HF-LPME procedure [34]. The technique is established the microextraction of CRTD and 1-octanol+1-undecanol (6:4, v:v) was used as extracting solvent. The results show that the method achieved low LODs and high recoveries of two CRTDs.

Vitamin E derivatives ( $\alpha-, \beta-, \gamma-$, and $\alpha$-TOC, and tocotrienols) and CRTD (LT, ZX, CRX, LYC, and $\beta$-CRT) in tomato fruits were determined simultaneously by a simple HPLC method [35]. Six different solvents (hexane, acetone, ethanol, methanol, ethyl acetate and tetrahydrofurane) were used individually or as a composition of equal ratios for extraction. The mixture of ethyl acetate/hexane $(1: 1, \mathrm{v} / \mathrm{v})$ showed the best result as the extraction solvent for tomato fruits. And LYC was discovered to be present in greatest amount in tomato pulp, followed by $\beta$-CRT and LT.

Sugars, organic acids, aroma components and CRTD in grapefruits were determined simultaneously by HPLC and GC-MS [36]. LT, ZX, $\beta$-CRX, LYC, $\alpha$-CRT and $\beta$-CRT were quantified in the tested grapefruit. $\beta$-CRT was the most abundant CRTD in these pulps and found $18.40-87.15 \%$ of the whole CRTs.

LT, $\beta$-CRX and $\beta$-CRT were evaluated from six freshwater algal species (Chlorella fusca, C. vulgaris, Selenastrum capricornutum, Pandorina morum, Botryococcus sudeticus and Chlorococcum sp.) using HPLC method [37]. The objective of this method is to investigate new resource of natural pigments to be use as food coloring agent. The results show that luteion was determined significantly higher in C.fusca $(69.54 \pm 11.29 \mu \mathrm{g} / \mathrm{g}) ; \beta-\mathrm{CRX}$ in P. morum $(1.24 \pm 0.33 \mu \mathrm{g} / \mathrm{g})$ though $\beta$-CRT in C. vulgaris $(18.42 \pm 9.2 \mu \mathrm{g} / \mathrm{g})$.

A validated RP-HPLC method for CRTD, chlorophylls and $\alpha$-TOC constituents of six vegetables was developed by Zeb [38]. Twelve CRTDs primarily LT and its three isomers, two cis-isomers of ZX, 8-apo-carotenal, alltrans- $\beta$-CRT and its 13 -cis-isomer; one fatty acid ester, $\alpha$-TOC and chlorophyll $\mathrm{a}$ and $\mathrm{b}$ were determined simultaneously in vegetable leaves. $\alpha$-TOC, neoxanthin, violaxanthin, LT, 8-apo-carotenal, chlorophyll a and all-trans- $\beta$-CRT were present in greatest amounts.

A validated multi-analyte method using with RP-HPLC for analysis of CRTDs used as feed additives in poultry and fish feed was developed by Vincent et.al. [39]. Ten CRTD (astaxanthin, astaxanthin dimethyldisuccinate, adonirubin, canthaxanthin, $\beta$-CRT, capsanthin, ethyl ester of beta-apo- $8^{\prime}$ carotenoic acid, citranaxanthin, LT and ZX) were determined in three stages; digestion of the feed sample with an enzyme, pressurised liquid extraction, and determination of the analytes by RP-HPLC with a PDA detector.

UPLC is a new method which works similarly with HPLC. CRTD pigments in pumpkin, honeybee pollen samples and nectarine flesh were determined by UPLC method. Analysis of seven CRTDs was completed in $8.5 \mathrm{~min}$ [40].

Because of its higher susceptibility and selectivity than traditional methods, mass spectrometric (MS) detection has fairly widespread use among researchers. CRTD esters in mandarin essential oil were determined by LC-APCI-MS. For the determination of free CRTD comparative retention times were used, UV absorptions and MS spectra with those of related standard. Superior resolution with the polymeric C-30 stationary phase provided a better ability to distinguish compared to traditional C-18 column. Based on high levels of CRX and its esters, mandarin peel can be considered as a good resource of vitamin A precursors [41].

Kruz et.al. [42] improved a HPLC assay with DAD and characterised by HPLC-APCI-MS for simultaneous separation of CRTDs and CRTD esters from six apricots. CRTDs were extracted with acetone/hexane or methanol and analytes were determined by HPLC and LC-MS without prior saponification. In apricots, $\alpha$-CRT was not detected. CRTD content of a yellow and red pepper during ripening, marketed in Campinas, Brazil was determined by HPLC method using DAD or MS detection. In the yellow pepper, the major CRTs were violaxanthin $(27.1-36.6 \mu \mathrm{g} / \mathrm{g})$, LT $(5.3-9.3 \mu \mathrm{g} / \mathrm{g}), \quad \beta$-CRT $(1.6-3.9 \mu \mathrm{g} / \mathrm{g})$. Neoxanthin, (Z)-violaxanthin, (Z)-antheraxanthin, (Z)-LT, $\alpha$-CRX, $\beta$-CRX, $\zeta$ CRT, $\alpha$-CRT and phytofluene were also investigated as subsidiary CRTDs. In red pepper, primary CRTDs were capsanthin $(29.2-36.2 \mu \mathrm{g} / \mathrm{g})$, LT (5.8-
$8.7 \mu \mathrm{g} / \mathrm{g}), \beta$-CRT $(5.1-6.8 \mu \mathrm{g} / \mathrm{g})$, and violaxanthin $(1.8-3.6 \mu \mathrm{g} / \mathrm{g})$. Minor CRTs were capsorubin, $\beta$-apo- $8^{\prime}$-carotenal, antheraxanthin, ZX, and $\beta$-CRX [43]. Quantification of $\beta$-CRT and LYC in tomatoes was achieved using TQMS in positive ionisation mode after extraction with ethyl acetate and hexane [44]. LYC and $\beta$-CRT contents were determined to be within the range of $47.1-88.5 \mathrm{mg} / \mathrm{kg}$ and $0.7-3.9 \mathrm{mg} / \mathrm{kg}$ respectively.

Giuffrida et.al. [45] improved a HPLC-DAD-APCI-MS technique for nonsaponified CRTD content in fresh peach fruits and market peach juice and jam. ZX, $\beta$-CRX, $\beta$-CRT and three monoesters of $\beta$-CRX were investigated for all the samples. All of them, $\beta$-CRT was the highest ratio in all samples found, with an approximate constituent of $1.61 \mu \mathrm{g} / \mathrm{g}$ and $1.42 \mu \mathrm{g} / \mathrm{g}$ in the market jam and juice and, respectively, $1.98 \mu \mathrm{g} / \mathrm{g}$ in the fresh fruits.

CRTDs in two different pumpkin species (Cucurbita maxima and C. moschata) were identified and quantified with a HPLC-UV/Vis-APCI-MS/MS technique by Bergantin et.al. [46]. The results show that the amount of LT and $\beta$-CRT was higher in C. maxima.

LC-ELSD is a useful technique for the quantification of CRTD in food samples. $\beta$-sitosterol, stigmasterol, campesterol, and $\alpha, \delta$ and $\gamma-$ TOC were determined by HPLC analysis based on ELSD while LT was determinated at 450 $\mathrm{nm}$ [47]. Implementation of ELSD enable quantification of sterols at the expense of loss in sensitivity. Addition of DAD to the system in line with ELSD is expected to increase the facility to determine all TOCs at levels existent in soybeans.

\section{CONCLUSION}

CRTD are natural colorants present in plants, such as vegetables and fruits having different colours from yellow to red. CRTDs importance in healthcare increased with clarification of their function as antioxidants, provitamin A, proliferation and cell differentiation regulators, cell-cell communication stimulators, carcinogen metabolism and immune function modulators and blue light filters. Because of CRTDs are fragile to light, temperature, oxygen and active areas, many of them are chemically similar and sensitive to isomerization and oxidation during analysis which complicate the analysis of these compounds. As a consequence of growing interest in the role of CRTD in healthcare, qualitative and quantitative analysis of CRTDs in food items became a hot topic. HPLC is the most preferred technique for the quantification of these phytochemicals especially the reversed-phase HPLC methods. With the use of the most recent techniques involving UPLC, analysis time shortened, solvent consumption decreased and better peak resolution was achieved. DAD was the preferred detector for quantification. Considering the subtle distinctness in significative UV-visible spectrum of many respective CRTDs the role of DAD is essential. Reversed phase separation are the most suitable systems and they are widely used with polymeric C-18 column for CRTD separation. Traditional C18 column has been predominated by the polymeric C-30 column in recent years. In spite of many solvent systems have been proposed as mobile phase for the analysis of CRTDs, the major solvents have been acetonitrile and methanol. Many of them were made simple modifications of some basic compositions. Including of trimethylamine in mobile phase which contains acetonitrile-based solvents was investigated to increase CRTD recovery. On the other side, a composition of methanol and methyl tert-butyl ether was used in the greater part of the systems that evaluated the C-30 column.

CRTDs are generally obtained from biological samples containing large quantities of water, with water-miscible organic solvent, for example acetone, ethanol, methanol, or mixtures thusly, to allow better solvent impression. Waterimmiscible solvents can be used for the extraction of dried materials, but in general the extraction is more impressive if the samples are rehydrated first and then extracted with water-miscible solvents. Acetone has been often used, but with coming of HPLC, tetrahydrofuran has also become a famous solvent for extraction.

A number of detection for instance UV, DAD, ELSD, MS, MS/MS are available for analysis of CRTD in food or biological matrices. LC/MS-MS performs a set of advantages over the conventional analytical methods, for instance improved selectivity, high sensibility and individuality, often with simple or without any sample preparation. But, chromatographic instrumentation in combination with MS-MS detection is very expensive and requires a speciality to operate. Therefore, there is a growing need to rapid, accurate and nondestructive techniques for the analysis of CRTDs in food matrices. 


\section{REFERENCES}

1. Krinsky, N.I. and E.J.J.M.a.o.m. Johnson, Carotenoid actions and their relation to health and disease. 2005. 26(6): p. 459-516.

2. Meléndez-Martínez, A.J., et al., Analysis of carotenoids in orange juice. 2007. 20(7): p. 638-649.

3. Rao, A.V. and L.G.J.P.r. Rao, Carotenoids and human health. 2007. 55(3): p. 207-216.

4. Gann, P.H., et al., Lower prostate cancer risk in men with elevated plasma lycopene levels: results of a prospective analysis. 1999. 59(6): p. 1225-1230.

5. Seddon, J.M., et al., Dietary carotenoids, vitamins A, C, and E, and advanced age-related macular degeneration. 1994. 272(18): p. 1413-1420.

6. IUPAC. IUPAC Carotenoid Nomenclature Rules,. Accessed 2011 [cited 2011 24.06.2011]; Available from: http://www.chem.qmul.ac.uk/iupac/carot/.

7. Britton, G., S. Liaaen-Jensen, and H. Pfander, Carotenoids: handbook. 2012: Birkhäuser.

8. Goodwn, T., The Biochemistry of the Carotenoids (Vol. 1: Plants). 1980, Chapman and Hall, London.

9. Aust, O., et al., Analysis of lipophilic antioxidants in human serum and tissues: tocopherols and carotenoids. 2001. 936(1-2): p. 83-93.

10. Barba, A.O., et al., Application of a UV-vis detection-HPLC method for a rapid determination of lycopene and $\beta$-carotene in vegetables. 2006. 95(2): p. $328-336$.

11. Hulshof, P.J., et al., Variation in retinol and carotenoid content of milk and milk products in The Netherlands. 2006. 19(1): p. 67-75.

12. Schlatterer, J., S. Maurer, and D.E.J.J.o.C.A. Breithaupt, Quantification of 3R, 3' R-zeaxanthin in plant derived food by a diastereomeric dilution assay applying chiral high-performance liquid chromatography. 2006. 1137(2): p. 216-222.

13. Kimura, M., et al., Screening and HPLC methods for carotenoids in sweetpotato, cassava and maize for plant breeding trials. 2007. 100(4): p. 1734-1746.

14. Wang, Y.-C., Y.-C. Chuang, and Y.-H.J.F.c. Ku, Quantitation of bioactive compounds in citrus fruits cultivated in Taiwan. 2007. 102(4): p. 1163-1171.

15. Marinova, D., F.J.J.o.F.C. Ribarova, and analysis, HPLC determination of carotenoids in Bulgarian berries. 2007. 20(5): p. 370-374

16. Gill, B.D. and H.E.J.I.d.j. Indyk, Liquid chromatographic method for the determination of lutein in milk and pediatric formulas. 2008. 18(9): p. 894898.

17. Dias, M.G., M.F.G. Camões, and L.J.F.C. Oliveira, Carotenoids in traditional Portuguese fruits and vegetables. 2009. 113(3): p. 808-815.

18. Sérino, S., et al., HPLC Assay of Tomato Carotenoids: Validation of a Rapid Microextraction Technique. Journal of Agricultural and Food Chemistry, 2009. 57(19): p. 8753-8760.

19. Aruna, G., B.S. Mamatha, and V. Baskaran, Lutein content of selected Indian vegetables and vegetable oils determined by HPLC. Journal of Food Composition and Analysis, 2009. 22(7): p. 632-636.

20. Liu, S.-C., J.-T. Lin, and D.-J. Yang, Determination of cis- and trans- $\alpha$ - and $\beta$-carotenoids in Taiwanese sweet potatoes (Ipomoea batatas (L.) Lam.) harvested at various times. Food Chemistry, 2009. 116(3): p. 605-610.

21. Dias, M.G., et al., Critical assessment of three high performance liquid chromatography analytical methods for food carotenoid quantification. 2010. 1217(21): p. 3494-3502.

22. Huang, X.-Y., et al., An Improved LC-DAD Method for Simultaneous Determination of Lutein, $\beta$-Carotene and Lycopene in Tomato and Its Products. Chromatographia, 2010. 71(3): p. 331-334.

23. Franke, S., et al., Analysis of carotenoids and vitamin E in selected oilseeds, press cakes and oils. European Journal of Lipid Science and Technology, 2010. 112(10): p. 1122-1129.

24. Gleize, B., et al., Simple and fast HPLC method for simultaneous determination of retinol, tocopherols, coenzyme Q10 and carotenoids in complex samples. Food Chemistry, 2012. 134(4): p. 2560-2564.

25. Cucu, T., et al., A Simple and Fast HPLC Method to Determine Lycopene in Foods. Food Analytical Methods, 2012. 5(5): p. 1221-1228.

26. Montesano, D., et al., A Simple and Selective Analytical Procedure for the Extraction and Quantification of Lutein from Tomato By-Products by HPLC-DAD. Food Analytical Methods, 2012. 5(4): p. 710-715.

27. Xiao, Z., et al., Assessment of Vitamin and Carotenoid Concentrations of Emerging Food Products: Edible Microgreens. Journal of Agricultural and Food Chemistry, 2012. 60(31): p. 7644-7651.

28. Simonovska, B., et al., Effects of extraction and high-performance liquid chromatographic conditions on the determination of lutein in spinach. Journal of Chromatography A, 2013. 1276: p. 95-101.
29. Ligor, M., et al., Study of RP HPLC Retention Behaviours in Analysis of Carotenoids. Chromatographia, 2014. 77(15): p. 1047-1057.

30. Gupta, P., Y. Sreelakshmi, and R. Sharma, A rapid and sensitive method for determination of carotenoids in plant tissues by high performance liquid chromatography. Plant Methods, 2015. 11(1): p. 5.

31. Górnaś, P., Unique variability of tocopherol composition in various seed oils recovered from by-products of apple industry: Rapid and simple determination of all four homologues $(\alpha, \beta, \gamma$ and $\delta)$ by RP-HPLC/FLD. Food Chemistry, 2015. 172: p. 129-134.

32. Bhatnagar-Panwar, M., et al., Rapid, accurate and routine HPLC method for large-scale screening of pro-vitamin A carotenoids in oilseeds. Journal of Plant Biochemistry and Biotechnology, 2015. 24(1): p. 84-92.

33. Kola, O., et al., HPLC determination of carotenoid, organic acid, and sugar content in pepino (Solanum muricatum) fruit during the ripening period. Chemistry of Natural Compounds, 2015. 51(1): p. 132-136.

34. Wang, J., Y. Yang, and N. Wu, Determination of Carotenoids in Egg Yolk by High Performance Liquid Chromatography with Vortex-Assisted Hollow Fiber Liquid-Phase Microextraction using Mixed Extraction Solvent. Journal of Chromatographic Science, 2016. 54(10): p. 1834-1840.

35. Irakli, M., et al., Optimization and development of a high-performance liquid chromatography method for the simultaneous determination of vitamin $\mathrm{E}$ and carotenoids in tomato fruits. 2016. 39(17): p. 3348-3356.

36. Zheng, H., et al., Determination of sugars, organic acids, aroma components, and carotenoids in grapefruit pulps. Food Chemistry, 2016. 205: p. 112-121.

37. Othman, R., et al., Determination of natural carotenoid pigments from freshwater green algae as potential halal food colorants. International Food Research Journal, (2017). 24, : p. 468-S471.

38. Zeb, A., A simple, sensitive HPLC-DAD method for simultaneous determination of carotenoids, chlorophylls and $\alpha$-tocopherol in leafy vegetables. Journal of Food Measurement and Characterization, 2017. 11(3): p. 979-986.

39. Vincent, U., F. Serano, and C. von Holst, Development and validation of a multi-analyte method for the regulatory control of carotenoids used as feed additives in fish and poultry feed. Food Additives \& Contaminants: Part A, 2017. 34(8): p. 1285-1297.

40. Bohoyo-Gil, D., et al., UHPLC as a suitable methodology for the analysis of carotenoids in food matrix. European Food Research and Technology, 2012. 235(6): p. 1055-1061.

41. Giuffrida, D., et al., Application of HPLC-APCI-MS with a C-30 reversed phase column for the characterization of carotenoid esters in mandarin essential oil. 2006. 21(2): p. 319-323.

42. Kurz, C., R. Carle, and A. Schieber, HPLC-DAD-MSn characterisation of carotenoids from apricots and pumpkins for the evaluation of fruit product authenticity. Food Chemistry, 2008. 110(2): p. 522-530.

43. de Azevedo-Meleiro, C.H. and D.B. Rodriguez-Amaya, Qualitative and quantitative differences in the carotenoid composition of yellow and red peppers determined by HPLC-DAD-MS. 2009. 32(21): p. 3652-3658.

44. Lucini, L., et al., Rapid determination of lycopene and $\beta$-carotene in tomato by liquid chromatography/electrospray tandem mass spectrometry. 2012 . 92(6): p. 1297-1303.

45. Giuffrida, D., et al., Determination of the carotenoid profile in peach fruits, juice and jam. 2013. 68(1): p. 39-44.

46. Bergantin, C., et al., HPLC-UV/Vis-APCI-MS/MS Determination of Major Carotenoids and Their Bioaccessibility from "Delica" (Cucurbita maxima) and "Violina" (Cucurbita moschata) Pumpkins as Food Traceability Markers. 2018. 23(11): p. 2791.

47. Slavin, M. and L. Yu, A single extraction and HPLC procedure for simultaneous analysis of phytosterols, tocopherols and lutein in soybeans. Food Chemistry, 2012. 135(4): p. 2789-2795. 